Bull. Austral. Math. Soc.

$57 \mathrm{R} 20,14 \mathrm{H} 45$

VoL. $62(2000)$ [177-182]

\title{
A REMARK ON THE $\eta$-INVARIANT FOR AUTOMORPHISMS OF HYPERELLIPTIC RIEMANN SURFACES
}

\author{
TAKAYUKI MORIFUJI
}

\begin{abstract}
We give a characterisation for the vanishing of the $\eta$-invariant of prime order automorphisms of hyperelliptic Riemann surfaces through the mapping torus construction. To this end, we introduce a notion of $s$-symmetry for finite order surface automorphisms.
\end{abstract}

\section{INTRODUCTION}

The $\eta$-invariant of the signature operator for an oriented closed Riemannian 3manifold was originally introduced by Atiyah, Patodi and Singer in the 1970's. As shown in [2], this can be regarded as the correction term of the Hirzebruch signature formula when one applies it to a Riemannian 4-manifold with boundary. That is to say, this invariant is equal to the integral of the first Pontrjagin form of a given Riemannian metric minus the signature of the bounding 4-manifold. Using this formula, explicit calculations of the $\eta$-invariant for various manifolds have been given by several authors.

In this note, we consider finite order mapping tori as our object. That is, given an automorphism of a closed oriented surface $T: F \rightarrow F$, we form the mapping torus $M_{T}$ by taking $F \times I$ and glueing $F \times\{0\}$ and $F \times\{1\}$ via $T$. This gives a surface bundle over $S^{1}$ whose diffeomorphism type is determined by its holonomy map, up to conjugacy and isotopy. When $T$ is of finite order, we endow $M_{T}$ with the metric which is induced from the product of the standard metric on $S^{1}$ and $T$-invariant metric of $F$, and fix it throughout this note. Here it should be noted that $\eta\left(M_{T}\right)$ is independent of the choice of invariant metric on $F$ (see $[8,9]$ ). Then we can regard the $\eta$-invariant of the mapping tori as a function

$$
\eta:\{\text { finite order automorphisms of } F\} \rightarrow \mathbb{Q}
$$

defined by $\eta(T)=\eta\left(M_{T}\right)$ (see the next section as to the rationality).

The purpose of this note is to give a topological description of the "kernel", which means the whole inverse image of zero, of the above function in the case where holonomies are hyperelliptic automorphisms of prime order. By definition, it is easy to see that if

Received 30th June, 1999

The author would like to express his gratitude to Professor Shigeyuki Morita for his encouragement. This research is supported in part by JSPS Research Fellowship for Young Scientists.

Copyright Clearance Centre, Inc. Serial-fee code: 0004-9727/00 \$A2.00+0.00. 
$M_{T}$ admits an orientation reversing isometry, then its $\eta$-invariant vanishes. However, it seems that other characterisations for the vanishing of the $\eta$-invariant are not well known. In the present note, we shall show that the kernel consists of automorphisms with s-symmetry (see Section 2 for the definition) under the above hypothesis. This can be regarded as a generalisation of our previous results (see $[10,11])$ to surfaces of higher genera. The precise statement and its proof are given in Section 3.

\section{Preliminaries}

We first review the fixed point data of automorphisms of an oriented closed surface $F$ (not a sphere) and the formula for the $\eta$-invariant of finite order mapping tori using it (see $[1,8]$ ). As for the definition of the $\eta$-invariant of the signature operator, see the original paper [2].

Let $T: F \rightarrow F$ be an orientation preserving diffeomorphism of finite order $m$. We denote the set of points of $F$ at which $G=\mathbb{Z} / m \mathbb{Z} \cong\langle T\rangle$ does not act freely by $\operatorname{Fix}(G)$. Let $\left\{x_{i}\right\}$ be a set of representatives of the orbits of $\operatorname{Fix}(G)$ under $G$ and $\alpha_{i}=\left|\operatorname{stab}_{G}\left(x_{i}\right)\right|$. Then $T^{m / \alpha_{i}}$ generates $\operatorname{stab}_{G}\left(x_{i}\right)$ so it acts faithfully by rotation on the tangent space at $x_{i}$. Let $\beta_{i}$ be an integer such that $T^{\beta_{i} m / \alpha_{i}}$ acts by rotation through $2 \pi \sqrt{-1} / \alpha_{i}$. The integer $\beta_{i}$ is well defined modulo $\alpha_{i}$ and $\left(\alpha_{i}, \beta_{i}\right)=1$, so that $\beta_{i} / \alpha_{i}$ is uniquely determined as an element of $\mathbb{Q} / \mathbb{Z}$. By the fixed point data of $T$, we mean the collection

$$
\sigma(T)=\left\langle g, m \mid \beta_{1} / \alpha_{1}, \cdots, \beta_{r} / \alpha_{r}\right\rangle,
$$

where $g$ is the genus of $F$ and $\beta_{i} / \alpha_{i} \in \mathbb{Q} / \mathbb{Z}$ are not ordered. Further the fixed point data satisfies the relation

$$
\sum_{k=1}^{r} \frac{\beta_{k}}{\alpha_{k}} \equiv 0(\bmod \mathbb{Z})
$$

By using the fixed point data, the $\eta$-invariant of the mapping torus $M_{T}$ constructed from $T$ is given by

$$
\eta\left(M_{T}\right)=-4 \sum_{k=1}^{r} s\left(\beta_{k}, \alpha_{k}\right),
$$

where $s\left(\beta_{k}, \alpha_{k}\right)$ is the Dedekind sum (see $\left.[1,8]\right)$. It should be remarked that the right hand side is rational and does not depend on the genus of $F$.

REMARK 2.1. The above formula is originally due to Meyerhoff and Ruberman [8, Theorem 1.3]. On the other hand, Akita shows in [1] that the same formula can be derived through a formula due to the author [9] by using the theory of automorphisms of a compact Riemann surface.

Next we recall well known notions in the Nielsen-Thurston theory of surface automorphisms (see [4]).

An essential 1-submanifold of $F$ is a disjoint union of simple closed curves in $F$ each component of which does not bound a 2-disk in $F$, and no two components of which are 
homotopic. An automorphism of $F$ is reducible if it leaves some essential 1-submanifold of $F$ invariant. An irreducible automorphism is one which is not reducible.

Moreover we define a notion of s-symmetry for finite order automorphisms.

DEFINITION 2.2: We say a finite order automorphism $T$ has s-symmetry if the cardinality of the singular locus of $F /\langle T\rangle$, denoted by $\nu(T)$, is not three.

REMARK 2.3. It should be noted that $\nu(T)$ coincides with the number of fixed points of $T$ when $T$ is of prime order. Further we easily notice by Gilman's result (see [6]) that s-symmetric automorphisms are reducible, but the converse does not hold in general. However if the genus of $F$ is equal to 1,2 or 3 , then reducibility implies s-symmetry (see [3]).

\section{Statement of the Result}

We are now in the position to state our result. A hyperelliptic involution $J$ of a surface $F$ is an involution on $F$ that fixes $2 g+2$ points. An automorphism $T$ is called hyperelliptic if it commutes with a fixed hyperelliptic involution $J$.

THEOREM 3.1. Let $T$ be a hyperelliptic automorphism of prime order of an oriented closed surface $F$. Then the following conditions are equivalent:

(i) $T \in \operatorname{Ker} \eta$ (namely, $\eta(T)=0$ holds).

(ii) $T$ has s-symmetry.

In particular if the genus of $F$ is 1,2 or 3, these two conditions hold if and only if $T$ is reducible.

Proof: When $F$ is a torus, we can easily check the theorem, because possible orders of $T$ are 2 or 3 (see [9, Example 3.3]).

We shall make use of the following lemma, which is shown by the standard results on the automorphism group of a compact Riemann surface (see [5], Chapter V).

LEMmA 3.2. Let $F$ be a hyperelliptic Riemann surface of genus $g \geqslant 2$ and $T \in$ Aut $F$ be of prime order $p$. Further we denote the genus of $F /\langle T\rangle$ by $\bar{g}$. Then the possibilities of the order of $T$, the number of fixed points and its branching data are given in Table 1.

In order to prove the theorem, we actually compute the $\eta$-invariant in each case of Lemma 3.2 by using the formula mentioned before.

First for involutions, our previous result says that the $\eta$-invariants in these cases always vanish (see [9, Example 3.2]).

Here, for later use, we review some basic properties of the Dedekind sum (see [12] for details).

Lemma 3.3. Let $\alpha, \beta, \beta^{\prime}$ be integers such that $(\alpha, \beta)=\left(\alpha, \beta^{\prime}\right)=1$. Then the Dedekind sum $s(\beta, \alpha)$ satisfies the following: 
Table 1: Prime order automorphisms of hyperelliptic Riemann surfaces

\begin{tabular}{c|c|c}
\hline Order $p$ & Number of fixed points $\nu(T)$ & Branching data \\
\hline 2 & 0 & $(\bar{g}:-)$ \\
2 & 2 & $(\bar{g}: 2,2)$ \\
2 & $2 g+2$ & $(2,2, \cdots, 2)$ \\
$g / \bar{g}$ & 2 & $(\bar{g}: p, p)$ \\
$(2 g+1) /(2 \bar{g}+1)$ & 3 & $(\bar{g}: p, p, p)$ \\
$(g+1) /(\bar{g}+1)$ & 4 & $(\bar{g}: p, p, p, p)$ \\
\hline
\end{tabular}

(i) If $\beta^{\prime} \equiv \pm \beta(\bmod \alpha)$, then $s\left(\beta^{\prime}, \alpha\right)= \pm s(\beta, \alpha)$.

(ii) If $\beta \beta^{\prime} \equiv 1(\bmod \alpha)$, then $s\left(\beta^{\prime}, \alpha\right)=s(\beta, \alpha)$.

(iii) (Reciprocity formula) $s(\beta, \alpha)+s(\alpha, \beta)=-1 / 4+1 / 12(\beta / \alpha+1 / \alpha \beta+\alpha / \beta)$.

Now for $p=g / \bar{g}$, since the number of fixed points of $T$ is two, we obtain the fixed point data $\left\langle g, g / \bar{g} \mid q_{1} / p, q_{2} / p\right\rangle$, where $\left(p, q_{i}\right)=1$ and $q_{1}+q_{2} \equiv 0(\bmod p)$. Hence using Lemma 3.3 (i), we can calculate as follows:

$$
\begin{aligned}
\eta(T) & =-4\left\{s\left(q_{1}, p\right)+s\left(q_{2}, p\right)\right\} \\
& =-4\left\{s\left(q_{1}, p\right)-s\left(q_{1}, p\right)\right\}=0
\end{aligned}
$$

In the case that $p=(2 g+1) /(2 \bar{g}+1)$, only one fixed point is the Weierstrass point and other two fixed points $x_{1}, x_{2}$ satisfy $x_{2}=J\left(x_{1}\right)$ (see [5]). Thus we have $\langle g, p|$ $q / p, q / p,(p-2 q) / p\rangle,(p, q)=1$ as the fixed point data. This is the $q^{*}$ th power of $\sigma(T)=$ $\langle g, p \mid 1 / p, 1 / p,(p-2) / p\rangle$, where $q q^{*} \equiv 1(\bmod p)$, so that it is sufficient to compute for $q=1$. In fact by means of Lemma 3.3 , we obtain

$$
\eta(T)=-\frac{(p-1)^{2}}{2 p}
$$

and this value is a nonzero rational number (see [1]). For the $q^{*}$ th power of $T$, using a formula of the $\eta$-invariant obtained in [9] we have

$$
\begin{aligned}
\eta\left(T^{q^{*}}\right) & =q^{*} \eta(T)-\sum_{k=1}^{q^{*}-1} \tau\left(T, T^{k}\right) \\
& \equiv-\frac{(p-1)^{2} q^{*}}{2 p}(\bmod \mathbb{Z})
\end{aligned}
$$

so that this is also nonzero. Here $\tau$ is Meyer's signature 2-cocycle (see [7]) of the mapping class group (namely, diffeomorphism group modulo the connected component of the identiy) of $F$. 
Finally when $\nu(T)=4$, all of them are non-Weierstrass points (see [5]), so that they are divided into two pairs which are interchanged by the hyperelliptic involution $J$. Therefore it follows that $\sigma(T)=\left\langle g, p \mid q_{1} / p, q_{1} / p, q_{2} / p, q_{2} / p\right\rangle$, where $\left(p, q_{i}\right)=1$ and $2 q_{1}+2 q_{2} \equiv 0(\bmod p)$. Accordingly we can apply the same computation as before:

$$
\eta(T)=-4\left\{2 s\left(q_{1}, p\right)+2 s\left(q_{2}, p\right)\right\}=0 .
$$

By virtue of the branching data in Table 1, we can immediately distinguish whether each automorphism has s-symmetry or not. Further it is clear from Remark 2.3 that the last statement of the theorem holds. Therefore the above calculations complete the proof of Theorem 3.1.

The next corollary claims that the $\eta$-invariant reflects the reducibility of finite order automorphisms even for higher genera (see $[\mathbf{1 0}, \mathbf{1 1}])$.

COROLlaRY 3.4. Let $T$ be a hyperelliptic automorphism of prime order. If $T$ is irreducible, then $\eta(T) \neq 0$ holds. Namely, $T \in \operatorname{Ker} \eta$ implies that $T$ is reducible.

REMARK 3.5. In the above corollary, the assumptions are essentially needed. In fact, Akita shows in [1] that there exists an irreducible automorphism $T$ such that $\eta(T)=0$.

\section{REFERENCES}

[1] T. Akita, (manuscript in preparation).

[2] M.F. Atiyah, V.K. Patodi and I.M. Singer, 'Spectral asymmetry and Riemannian geometry I', Math. Proc. Camb. Phil. Soc. 78 (1975), 43-69.

[3] S.A. Broughton, 'Classifying finite group actions on surfaces of low genus', J. Pure Appl. Algebra 69 (1990), 233-270.

[4] A.J. Casson and S.A. Bleiler, Automorphisms of surfaces after Nielsen and Thurston (Cambridge Univ. Press, Cambridge, New York, 1988).

[5] H.M. Farkas and I. Kra, Riemann surfaces, Graduate Texts in Mathematics 71 (Springer-Verlag, New York, 1980).

[6] J. Gilman, 'Structures of elliptic irreducible subgroups of the modular group', Proc. London Math. Soc. (3) 47 (1983), 27-42.

[7] W. Meyer, 'Die Signatur von Flächenbündeln', Math. Ann. 201 (1973), 239-264.

[8] R. Meyerhoff and D. Ruberman, 'Cutting and pasting and the $\eta$-invariant', Duke Math. J. 61 (1990), 747-761.

[9] T. Morifuji, 'The $\eta$-invariant of mapping tori with finite monodromies', Topology Appl. 75 (1997), 41-49.

[10] T. Morifuji, 'On the reducibility and the $\eta$-invariant of periodic automorphisms of genus 2 surface', J. Knot Theory Ramifications 6 (1997), 827-831.

[11] T. Morifuji, 'A note on the reducibility of automorphisms of the Klein curve and the $\eta$-invariant of mapping tori', Proc. Amer. Math. Soc. 126 (1998), 1945-1947.

[12] H. Rademacher and E. Grosswald, Dedekind sums, Carus Mathematical Monographs 16 (Math. Assoc. Amer., Washington D.C., 1971). 
Graduate School of Mathematical Sciences

The University of Tokyo

Komaba, Meguro

Tokyo 153-8914

Japan

e-mail: morifuji@ms406ss5.ms.u-tokyo.ac.jp 